\title{
The Principle of Mathematical Induction Applied to the Generalized Model for the Economic Design of $\bar{x}$-Control Charts
}

\author{
Mohamed E. Seliaman ${ }^{1}$, Salih O. Duffuaa ${ }^{2}$ \\ ${ }^{1}$ Department of Information Systems, King Faisal University, Al-Ahsa, Saudi Arabia \\ ${ }^{2}$ Department of Systems Engineering, King Fahd University of Petroleum \& Minerals, Dhahran, Saudi Arabia \\ Email: mseliaman@gmail.com
}

Received August 4, 2012; revised September 6, 2012; accepted September 25, 2012

\begin{abstract}
Rahim and Banerjee [1] developed a general model for the optimal design of $\bar{x}$-control charts. The model minimizes the expected cost per unit time. The heart of the model is a theorem that derives the expected total cost and the expected cycle length. In this paper an alternative simple proof for the theorem is provided based on mathematical induction.
\end{abstract}

Keywords: Discrete Mathematics; Mathematical Induction; Statistical Quality Control; Design of $\bar{x}$-Control Charts; Hazard Rate Function

\section{Introduction}

Quality control charts are graphical statistical tools used for process control. The first control chart was developed by Walter A. Shewart [2]. Since then, these charts have been widely applied in industry and also received intensive attention from researchers. The $\bar{x}$-control charts are the most used statistical control charts when continuous variables are used to measure quality characteristics [3]. Generally, control charts are designed using four approaches: heuristics, statistical design, economic design and economic statistical design [4]. For more information on the design of control charts, interested readers are referred to [1-7].

The economic design of $\bar{x}$-control charts was originated by Duncan [8]. In his model, Duncan considers a production process that is subject to an occurrence of an assignable cause that will drive the process out of control. The output of the process is measurable on a certain scale and is normally distributed with mean $\mu_{0}$ and standard deviation $\sigma_{0}$. The assignable cause is assumed to occur according to a Possion process with intensity $\lambda$ and causes a shift in the process mean to $\mu_{0} \pm \delta$, where $\delta$ is a positive parameter. The control limits of the $\bar{x}$-control chart are set at $\mu_{0} \pm k \sigma_{0}$, where $\sigma_{0}$ is the standard deviation of the process. A sample of size $n$ is taken from the output of the process every $h$ hour, and used to decide whether the process is in control or not.

Banerjee and Rahim [9] generalized Duncan's model [8] for the economic design of $\bar{x}$-control charts by relaxing
Duncan's assumption that the in-control period follows an exponential distribution. Instead, they assumed it follows a general probability distribution having an increasing hazard rate function. The increasing hazard rate assumption resulted in the modification of the fixed sampling interval to a variable sampling interval, dependent on the process age. In both models, if the sample falls outside the control limits, a search is initiated to locate the assignable cause. If the search indicates that there is a false alarm, the process continues. On the other hand, if the alarm is true, repair or replacement is undertaken to bring the process in control. Later, Rahim and Banerjee [1] extended the model in Banerjee and Rahim [9] by assuming a general distribution of in-control periods with increasing failure rate and considering an age-dependent repair before failure. The objective of Rahim and Banerjee's model [1] is to find the parameters $n, h_{j}$, and $k$. Under the assumptions described for this generalized model, they derived expressions for the expected cycle length and the total expected cost per cycle. They also presented proofs for these expressions using a recursive argument. In this paper a simpler and shorter mathematical induction proof of these results is presented. Thus, our alternative proof may make it easier for students and professors interested in the topic within graduate coursework or further research on extending the model. In this paper, we adopt the same notations and model descriptions as defined in Rahim and Banerjee [1].

In order to make the paper self-contained, we describe the main elements involved in the design of control charts, 
as modeled in [1]

\section{Model Development}

In [1], the following assumptions are made:

1) The duration of the in-control period is assumed to follow an arbitrary probability density function, $f(t)$, having an increasing hazard rate, $r(t)$, and $F(t)$ as its cumulative density function.

2) The process is monitored by drawing random samples of size $n$ at times $h_{1}, h_{1}+h_{2}, h_{1}+h_{2}+h_{3} \cdots$ and so on. Further, $h_{j}$ satisfies: (i) $h_{1} \geq h_{2} \geq h_{3} \cdots$, and (ii) $\lim _{m \rightarrow \infty} F\left(\omega_{m}\right)=1$.

3) A production cycle begins with a new machine and ends either with a true alarm or at time $\omega_{m}$, whichever occur first. In other words, if no true alarm is observed by the time $\omega_{m-1}$, then the cycle is allowed to continue for an additional time $h_{m}$; at time $\omega_{m}$, the old component is replacement by a new one. Thus, there is no cost of sampling and charting during the $m^{\text {th }}$-sampling interval.

4) For mathematical simplicity, it is assumed that the production ceases during search and repair.

In their paper, Rahim and Banerjee [1] state the following theorem and provide a proof for it based on recursive relationships. Below, we first present their result and then provide a simpler mathematical induction proof.

\section{Theorem 1}

Under the assumptions (1)-(3) described above the following is true:

$$
\begin{aligned}
E(T) & =\sum_{j=1}^{m} h_{j} \bar{F}\left(\omega_{j-1}\right)+\alpha Z_{0} \sum_{j=1}^{m-1} \bar{F}\left(\omega_{j}\right) \\
& +\beta \sum_{j=1}^{m-1} \nabla F\left(\omega_{j}\right) \sum_{i=j+1}^{m} h_{i} \beta^{i-j-1}+Z_{1}
\end{aligned}
$$

and

$$
\begin{aligned}
& E(C)=(a+b n)\left[1+\sum_{j=1}^{m-2} \bar{F}\left(w_{j}\right)+\beta \sum_{j=1}^{m-2} \nabla F\left(\omega_{j}\right)\right. \\
& \left.\cdot\left\{(1-\beta) \sum_{i=1}^{m-1-j} i \beta^{i-1}+(m-1-j) \beta^{m-1-j}\right\}\right] \\
& +\left(D_{0}-D_{1}\right) \int_{0}^{\omega_{m}} x f(x) \mathrm{d} x+\left(D_{1}-D_{0}\right) \sum_{j=1}^{m} \omega_{j} \nabla F\left(\omega_{j}\right) \\
& +D_{0} \sum_{j=1}^{m} h_{j} \bar{F}\left(\omega_{j-1}\right)+W+\beta D_{1} \sum_{j=1}^{m-1} \nabla F\left(\omega_{j}\right)\left\{\sum_{i=j+1}^{m} \beta^{i-j-1} h_{i}\right\} \\
& +\alpha Y \sum_{j=1}^{m-1} \bar{F}\left(\omega_{j}\right)+\bar{F}\left(\omega_{m}\right) S\left(\omega_{m}\right) .
\end{aligned}
$$

\section{The Mathematical Induction Proof}

In this section, an alternative proof is provided for Theorem 1. The proof consists of the two well-known induction steps: the basis step and the inductive step. For this purpose, we consider the case after the first sampling interval as a new model with a smaller number of intervals and as having a new density function for the incontrol period.

\section{1) Expected cycle length:}

a) Let $\mathrm{M}$ be a model with one interval. Thus, $\mathrm{E}(\mathrm{T})=h_{1}+$ $\mathrm{Z}_{1}$, obtained by letting $m=1$ in Equation (1) (the basis step).

b) Assume that (1) is true for a model with a number of intervals less than $m$. Then we show that it is true for a model with $m$ intervals (the inductive step).

As in [7], let us view the possible states of the system at the end of the first sampling interval. For each possible state of the system, the expected residual times in the cycle and the associated probabilities are presented in

\section{Table 1.}

$E\left(T^{*}\right)$ is the expected cycle length for a model $\mathrm{M}^{*}$ with the following data.

1) $\mathrm{M}^{*}$ has $m-1$ intervals with $h_{j}^{*}=h_{j+1}$ for $j=1$, $2, \cdots, m-1$.

2) In $\mathrm{M}^{*}$, the duration of the in-control period is assumed to follow an arbitrary probability density function, $f^{*}(t)$, and $F^{*}(t)$ as its cumulative density function where

$$
f^{*}(t)= \begin{cases}o & t<\omega_{1} \\ \frac{f(t)}{1-p_{1}} & \omega_{1} \leq t,\end{cases}
$$

and $E\left(T^{* *}\right)$ is the expected cycle length for a model $M^{* *}$ with the following data.

1. $M^{* *}$ has $m-2$ intervals with $h_{j}^{* *}=h_{j+2}$ for $j=1,2, \cdots, m-2$.

2. In $M^{* *}$, the duration of the in-control period is assumed to follow an arbitrary probability density function, $f^{* *}(t)$, and $F^{* *}(t)$ as its cumulative density function where

$$
f^{* *}(t)= \begin{cases}\frac{f(t)}{p_{1}} & t \leq \omega_{1} \\ 0 & \omega_{1}<t .\end{cases}
$$

Table 1. The expected residual times.

\begin{tabular}{lcc}
\hline State & Probability & $\begin{array}{c}\text { Expected } \\
\text { residual time }\end{array}$ \\
\hline In control and no alarm & $\left(1-p_{1}\right)(1-\alpha)$ & $E\left(T^{*}\right)$ \\
In control and false alarm & $\left(1-p_{1}\right) \alpha$ & $Z_{0}+E\left(T^{*}\right)$ \\
Out of control but no alarm & $p_{1} \beta$ & $\mathrm{h}_{2}+E\left(T^{* *}\right)$ \\
Out of control and true alarm & $p_{1}(1-\beta)$ & $Z_{1}$ \\
\hline
\end{tabular}


Thus,

$$
\begin{aligned}
E(T) & =h_{1}+\left(1-p_{1}\right)(1-\alpha) E\left(T^{*}\right)+\left(1-p_{1}\right) \alpha\left[Z_{0}+E\left(T^{*}\right)\right]+p_{1} \beta\left[h_{2}+E\left(T^{* *}\right)\right]+p_{1}(1-\beta) Z_{1} \\
& =h_{1}+\left(1-p_{1}\right) E\left(T^{*}\right)+\left(1-p_{1}\right) \alpha Z_{0}+p_{1} \beta E\left(T^{* *}\right)+p_{1} \beta+h_{2}+p_{1}(1-\beta) Z_{1}
\end{aligned}
$$

Thus, according to the induction hypothesis along with the relations (3) and (4)

$$
\begin{aligned}
& E\left(T^{*}\right)=\sum_{j=1}^{m-1} h_{j}^{*} \bar{F}^{*}\left(\omega^{*}{ }_{j-1}\right)+\alpha Z_{0} \sum_{j=1}^{m-2} \bar{F}^{*}\left(\omega_{j}\right)+\beta \sum_{j=1}^{m-2} \nabla F^{*}\left(\omega_{j}^{*}\right) \sum_{i=j+1}^{m-1} h_{i}^{*} \beta^{i-j-1}+Z_{1} \\
& =\sum_{j=2}^{m} h_{j} \frac{\bar{F}\left(\omega_{j-1}\right)}{1-p_{1}}+\alpha Z_{0} \sum_{j=2}^{m-1} \frac{\bar{F}\left(\omega_{j}\right)}{1-p_{1}}+\beta \sum_{j=2}^{m-1} \nabla \frac{F\left(\omega_{j}\right)}{1-p_{1}} \sum_{i=j+1}^{m} h_{i} \beta^{i-j-1}+Z_{1},
\end{aligned}
$$

and

$$
E\left(T^{* *}\right)=\sum_{j=1}^{m-2} h_{j}^{* *} \bar{F}^{* *}\left(\omega_{j-1}\right)+\alpha Z_{0} \sum_{j=1}^{m-3} \bar{F}^{* *}\left(\omega_{j}^{* *}\right)+\beta \sum_{j=1}^{m-3} \nabla F^{* *}\left(\omega_{j}\right) \sum_{i=j+1}^{m-2} h_{i}^{* *} \beta^{i-j-1}+Z_{1}=Z_{1} .
$$

Substituting (6) and (7) into (5), we obtain

$$
\begin{aligned}
E(T) & =h_{1}+\sum_{j=2}^{m} h_{j} \bar{F}\left(\omega_{j-1}\right)+\left(1-p_{1}\right) \alpha Z_{0}+\alpha Z_{0} \sum_{j=2}^{m-1} \bar{F}\left(\omega_{j}\right)+p_{1} \beta h_{2}+\beta \sum_{j=2}^{m-1} \nabla F\left(\omega_{j}\right) \sum_{i=j+1}^{m} h_{i} \beta^{i-j-1}+Z_{1} \\
& =\sum_{j=1}^{m} h_{j} \bar{F}\left(\omega_{j-1}\right)+\alpha Z_{0} \sum_{j=1}^{m-1} \bar{F}\left(\omega_{j}\right)+\beta \sum_{j=1}^{m-1} \nabla F\left(\omega_{j}\right) \sum_{i=j+1}^{m} h_{i} \beta^{i-j-1}+Z_{1},
\end{aligned}
$$

which gives the proof for Equation (1) in the theorem.

\section{2) The Expected Total Cost}

To obtain the expected total cost $E(C)$, we consider the expected residual cost beyond time $h_{1}$ as the expected total cost for a model with less than $m$ intervals. For each possible state of the system at the end of the first sampling interval, the expected residual costs in the cycle and the associated probabilities are presented in Table 2.

Where $E\left(C^{*}\right)$ and $E\left(C^{* *}\right)$ are the two expected total costs for $M^{*}$ and $M^{* *}$ respectively, from the above table we obtain

$$
\begin{aligned}
E(C) & =\left(1-p_{1}\right)\left\{(a+b n)+D_{0} h_{1}+\alpha Y+\left(C^{*}\right)\right\} \\
& +{ }_{1}\left\{(a+b n)+{ }_{0} \tau_{1}+D_{1}\left(h_{1}-\tau_{1}\right)\right\} \\
& +p_{1} \beta D_{1} h_{2}+p_{1} \beta E\left(C^{* *}\right)+p_{1}(1-\beta) W .
\end{aligned}
$$

Thus, employing the induction hypothesis along with the relations (3) and (4),

$$
\begin{aligned}
E\left(C^{*}\right) & =(a+b n)\left[1+\sum_{j=2}^{m-2} \frac{\bar{F}\left(\omega_{j}\right)}{1-p_{1}}+\beta \sum_{j=2}^{m-2} \frac{\nabla F\left(\omega_{j}\right)}{1-p_{1}}\left\{(1-\beta) \sum_{i=1}^{m-1-j} i \beta^{i-1}+(m-1-j) \beta^{m-1-j}\right\}\right] \\
& +\left(D_{0}-D_{1}\right) \int_{w_{1}}^{w_{m}} \frac{x f(x) \mathrm{d} x}{1-p_{1}}+\left(D_{1}-D_{0}\right) \sum_{j=2}^{m} \omega_{j} \frac{\nabla F\left(\omega_{j}\right)}{1-p_{1}}+D_{0} \sum_{j=2}^{m} h_{j} \frac{\bar{F}\left(\omega_{j-1}\right)}{1-p_{1}}+W \\
& +\beta D_{1} \sum_{j=2}^{m-1} \frac{\nabla F\left(\omega_{j}\right)}{1-p_{1}}\left\{\sum_{i=j+1}^{m} \beta^{i-j-1} h_{i}\right\}+\alpha Y \sum_{j=2}^{m-1} \frac{\bar{F}\left(\omega_{j}\right)}{1-p_{1}}-\frac{\bar{F}\left(\omega_{m}\right)}{1-p_{1}} S\left(\omega_{m}\right)
\end{aligned}
$$

and

$$
E\left(C^{* *}\right)=(a+b n)+W
$$

Substituting (9) and (10) into (8), we obtain 
Table 2. The expected residual costs.

\begin{tabular}{llll}
\hline State & Probability & Current Cost & Expected Residual Cost \\
\hline In control and no alarm & $\left(1-p_{1}\right)(1-\alpha)$ & $(a+b n)+D_{0} h_{1}$ & $E\left(C^{*}\right)$ \\
In control and false alarm & $\left(1-p_{1}\right) \alpha$ & $(a+b n)+Y+D_{0} h_{1}$ & $E\left(C^{*}\right)$ \\
Out of control but no alarm & $p_{1} \beta$ & $(a+b n)+D_{0} \tau_{1}+D_{1}\left(h_{1}-\tau_{1}\right)$ & $D_{1} h_{2}+E\left(C^{* *}\right)$ \\
Out of control and true alarm & $p_{1}(1-\beta)$ & $(a+b n)+D_{0} \tau_{1}+D_{1}\left(h_{1}-\tau_{1}\right)$ & $W$ \\
\hline
\end{tabular}

$$
\begin{aligned}
E(C)= & (a+b n)\left[1+\sum_{j=1}^{m-2} \bar{F}\left(\omega_{j}\right)+\beta \sum_{j=1}^{m-2} \nabla F\left(\omega_{j}\right)\left\{(1-\beta) \sum_{i=1}^{m-1-j} i \beta^{i-1}+(m-1-j) \beta^{m-1-j}\right\}\right] \\
& +\left(D_{0}-D_{1}\right) \tau_{1}+\left(D_{0}-D_{1}\right) \int_{\omega_{1}}^{\omega_{m}} x f(x) \mathrm{d} x+p_{1} D_{1} h_{1}+\left(D_{1}-D_{0}\right) \sum_{j=2}^{m} \omega_{j} \nabla F\left(\omega_{j}\right) \\
& +\left(1-p_{1}\right) D_{0} h_{1}+D_{0} \sum_{j=2}^{m} h_{j} \bar{F}\left(\omega_{j-1}\right)+W+\beta D_{1} p h_{2}+\beta D_{1} \sum_{j=2}^{m-1} \nabla F\left(\omega_{j}\right)\left\{\sum_{i=j+1}^{m} \beta^{i-j-1} h_{i}\right\} \\
& +\alpha Y \sum_{j=1}^{m-1} \bar{F}\left(\omega_{j}\right)-\bar{F}\left(\omega_{m}\right) S\left(\omega_{m}\right) .
\end{aligned}
$$

By adding and subtracting the term $p_{1} D_{0} h_{1}$ to the right hand side of (11) and substituting

$$
\tau_{1}=\int_{\omega_{1}}^{\omega_{m}} x f(x) \mathrm{d} x,
$$

we obtain

$$
\begin{aligned}
E(C) & =(a+b n)\left[1+\sum_{j=1}^{m-2} \bar{F}\left(\omega_{j}\right)+\beta \sum_{j=1}^{m-2} \nabla F\left(\omega_{j}\right)\left\{(1-\beta) \sum_{i=1}^{m-1-j} i \beta^{i-1}+(m-1-j) \beta^{m-1-j}\right\}\right] \\
& +\left(D_{0}-D_{1}\right) \int_{0}^{\omega_{m}} x f(x) \mathrm{d} x+\left(D_{1}-D_{0}\right) \sum_{j=1}^{m} \omega_{j} \nabla F\left(\omega_{j}\right)+D_{0} \sum_{j=1}^{m} h_{j} \bar{F}\left(\omega_{j-1}\right)+W \\
& +\beta D_{1} \sum_{j=1}^{m-1} \nabla F\left(\omega_{j}\right)\left\{\sum_{i=j+1}^{m} \beta^{i-j-1} h_{i}\right\}+\alpha Y \sum_{j=1}^{m-1} \bar{F}\left(\omega_{j}\right)+\bar{F}\left(\omega_{m}\right) S\left(\omega_{m}\right) .
\end{aligned}
$$

This completes the proof.

\section{Acknowledgements}

The authors would like to acknowledge the support for this research provided by the King Fahd University of Petroleum and Minerals and King Faisal University.

\section{REFERENCES}

[1] M. A. Rahim and P. K. Banerjee, "A Generalized Model for the Economic Design of $\bar{x}$-Control Charts for Production Systems with Increasing Failure Rate and Early Replacement," Naval Research Logistics, Vol. 40, No. 6, 1993, pp. 787-809.

doi:10.1002/1520-6750(199310)40:6<787::AID-NAV322 $\underline{0400605>3.0 . C O ; 2-4}$
[2] D. C. Montgomery, "The Economic Design of Control Charts: A Review and Literature Survey," Journal of Quality Technology, Vol. 12, No. 2, 1980, pp. 75-87.

[3] D. Patel, "Economic Design of Control Chart," B. Tech Thesis, National Institute of Technology, Rourkela, 2009.

[4] R.-C. Wang and C.-H. Chen, "Economic Statistical NpControl Chart Designs Based on Fuzzy Optimization," International Journal of Quality \& Reliability Management, Vol. 12, No. 1, 1995, pp. 82-92. doi:10.1108/02656719510076276

[5] Y.-S. Chen and Y.-M. Yang, “An Extension of Banerjee and Rahim's Model for Economic Design of Moving Average Control Chart for a Continuous Flow Process," European Journal of Operational Research, Vol. 143, No. 3, 2002, pp. 600-610. doi:10.1016/S0377-2217(01)00341-1

[6] I. N. Gibra, "Economically Optimal Determination of the 
Parameters of $\bar{x}$-Control Chart," Management Science, Vol. 17, No. 9, 1971, pp. 635-646.

[7] E. M. Saniga, "Economic Statistical Control-Chart Designs with an Application to X and R Charts," Technometrics, Vol. 31, No. 3, 1989, pp. 313-320.

[8] A. J. Duncan, "The Economic Design of $\bar{x}$-Control Charts Used to Maintain Current Control of a Process,"
Journal of the American Statistics Association, Vol. 51, 1956, pp. 228-242.

[9] P. K. Banerjee and M. A. Rahim, "Economic Design of $\bar{x}$-Control Charts under Weibull Shock Models," Technometrics, Vol. 30, No. 4, 1998, pp. 407-414.

\section{Notation}

$Z_{0}=$ expected search time associated with a false alarm.

$Z_{1}=$ expected search time to discover the assignable cause and repair.

$a=$ fixed sampling cost.

$b=$ sampling cost per unit sampled.

$Y=$ cost per false alarm.

$W=$ cost to locate and repair the assignable cause.

$D_{0}=$ quality cost per hour while producing and the process is in control.

$D_{1}=$ quality cost per hour while producing and the process is out of control.

$\alpha=$ probability of Type I error. $\beta=$ probability of Type II error.

$h=$ the sampling interval for a uniform sampling scheme.

$h_{j}=$ the $j^{\text {th }}$ sampling interval for a non-uniform sampling scheme.

$\omega_{j}=h_{1}+h_{2}+\cdots+h_{\mathrm{j}}$.

$\mathrm{M}=$ a specified number of sampling intervals.

$n=$ sample size.

$E(T)=$ expected cycle length.

$E(C)=$ expected cost.

$P_{j}=$ the conditional probability that process goes out of control between $\omega_{j-1}$ and $\omega_{j}$, given that it was at the in-control state at time $\omega_{j-1}$. 